\title{
Medication errors in paediatric care: a systematic review of epidemiology and an evaluation of evidence supporting reduction strategy recommendations
}

\author{
Marlene R Miller, Karen A Robinson, Lisa H Lubomski, Michael L Rinke, Peter J Pronovost
}

Qual Saf Health Care 2007;16:116-126. doi: 10.1136/qshc.2006.019950

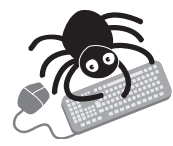

Appendices are available on the QSHC website http:// qshc.bmi.com/ supplemental

See end of article for authors' affiliations

Correspondence to:

Dr Marlene R Miller, Director

of Quality and Safety

Initiatives, Johns Hopkins

Children's Center, CMSC

2-125, $600 \mathrm{~N}$ Wolfe Street, Baltimore, MD 21287, USA; mmille21@jhmi.edu

Accepted 27 January 2007
Background: Although children are at the greatest risk for medication errors, little is known about the overall epidemiology of these errors, where the gaps are in our knowledge, and to what extent national medication error reduction strategies focus on children.

Objective: To synthesise peer reviewed knowledge on children's medication errors and on recommendations to improve paediatric medication safety by a systematic literature review.

Data sources: PubMed, Embase and Cinahl from 1 January 2000 to 30 April 2005, and 11 national entities that have disseminated recommendations to improve medication safety.

Study selection: Inclusion criteria were peer reviewed original data in English language. Studies that did not separately report paediatric data were excluded.

Data extraction: Two reviewers screened articles for eligibility and for data extraction, and screened all national medication error reduction strategies for relevance to children.

Data synthesis: From 358 articles identified, 31 were included for data extraction. The definition of medication error was non-uniform across the studies. Dispensing and administering errors were the most poorly and non-uniformly evaluated. Overall, the distributional epidemiological estimates of the relative percentages of paediatric error types were: prescribing 3-37\%, dispensing 5-58\%, administering 72-75\%, and documentation 17-21\%. 26 unique recommendations for strategies to reduce medication errors were identified; none were based on paediatric evidence.

Conclusions: Medication errors occur across the entire spectrum of prescribing, dispensing, and administering, are common, and have a myriad of non-evidence based potential reduction strategies. Further research in this area needs a firmer standardisation for items such as dose ranges and definitions of medication errors, broader scope beyond inpatient prescribing errors, and prioritisation of implementation of medication error reduction strategies.
$\mathrm{T}$ he Institute of Medicine report To Err Is Human shone a spotlight on preventable medical errors and since the release of the report patient safety has become the preeminent issue for health care. ${ }^{1}$ With our understanding of the problems and solutions for patient safety growing daily, it has become clear that the prescribing, dispensing, and administration of medications represent a substantial portion of the preventable medical errors that occur with children and that children are more at risk for medication errors than adults. ${ }^{23}$

Despite the awareness that children are at increased risk for medication errors, little is known about the epidemiology of these errors and where the gaps remain in our present knowledge. We conducted a systematic literature review to synthesise all the peer reviewed knowledge on medication errors for children published since the release of the To Err Is Human report. ${ }^{1}$ Our scope included all care settings and all types of medications. In addition, we synthesised all the recommendations to improve paediatric medication safety from national entities and evaluated the paediatric evidence provided to support these recommendations for effectiveness, efficacy, cost effectiveness, feasibility, appropriateness in different settings and institutional barriers.

\section{METHODS}

Study inclusion criteria for systematic literature review on medication errors

Articles eligible for inclusion in our synthesis had to report peer reviewed English language original data on the epidemiology of medication errors in children published between 1 January 2000 and 30 April 2005. Medication errors were defined as any preventable error in the medication administration process starting from prescribing and including preparing, dispensing, administering, monitoring the patient for effect, and transcribing (eg, medication administration record (MAR)). We only included adverse drug events (ADEs) that were described by the studies as either preventable or having significant potential for harm to the patient (fig 1). ${ }^{2}$

\section{Search strategy for systematic literature review on} medication errors

We completed searches of PubMed, Embase and Cinahl in April 2005. The search strategy combined terms for the population (eg, paediatric) and terms to identify articles dealing with medication errors (eg, medication errors as Medical Subject Heading, preventable adverse event) (Appendix l, available at http://qshc.bmj.com/supplemental). References for all eligible articles were also reviewed. The search results were tracked in a database created in the bibliographic software ProCite (ISI, Berkley, California, USA).

Two independent non-blinded reviewers screened the title and abstract of each article to determine eligibility. At the fulltext level, two non-blinded reviewers screened articles and, if the article was eligible, extracted relevant information in a

Abbreviations: ADEs, adverse drug events; MAR, medication administration record 


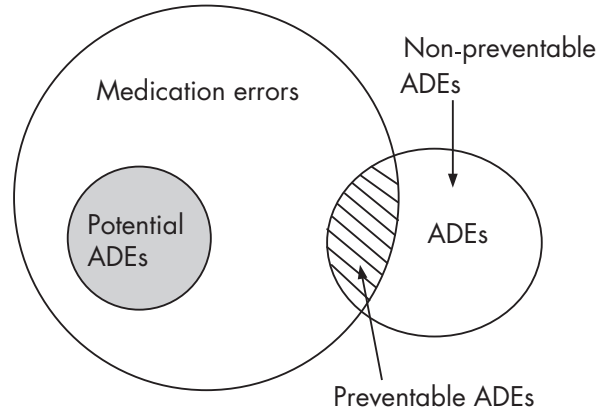

Figure 1 Relationship between medication errors, potential adverse drug events (ADEs), and ADEs. ${ }^{4}$

sequential fashion so that the second reviewer was able to see the extraction results from the first reviewer. All reviewers had either clinical degrees or health services research degrees with experience in systematic reviews. All reviewer pairings at all stages of this effort included at least one clinician, and the reviewer pairings for the abstracts were kept for the full text review. Discrepancies were resolved by consensus between the two reviewers after discussion. We developed and pilot tested forms to extract information such as duration of the study, type of study, the incidence of medication errors and information about the medication errors studied, such as the type and severity (Appendix 2, available at http://qshc.bmj.com/ supplemental). Evidence tables summarising the information from the articles were created from the spreadsheets and we qualitatively synthesised the literature since no articles included comparable numerators, denominators, or definitions for medication error that would have permitted quantitative synthesis of the articles.

\section{Synthesis of recommendations to reduce medication errors for children}

Working in conjunction with the Institute of Medicine, we identified national entities that have created and disseminated recommendations to improve medication safety either specifically for children or more broadly for all patients. These entities were: Institute for Safe Medication Practices, Pediatric Pharmacy Advocacy Group, American Hospital Association, American Academy of Pediatrics/National Initiative for Children's Healthcare Quality, Institute of Medicine, National Quality Forum, Massachusetts Hospital Association/ Massachusetts Coalition for the Prevention of Medical Errors, National Coordinating Council for Medication errors Reporting and Prevention, Agency for Healthcare Research and Quality, and Joint Commission on Accreditation of Healthcare Organizations. ${ }^{14-22}$ We reviewed all the published recommendations from these bodies and any cited literature to support the recommendations to determine whether this literature included, or was specific for, children.

\section{RESULTS}

\section{Literature search for systematic review on medication} errors

Our search identified 358 articles. Eight-four (23\%) of these articles were deemed eligible through the title and abstract screening. The most common reason for excluding an article from further consideration was lack of original data. A further 52 articles were excluded during the full-text review, and we were unable to retrieve one article, leaving 31 articles for full text data extraction..$^{23-53}$ Figure 2 provides an overview of the search and screening process (Appendix 3 lists the articles excluded, available at http://qshc.bmj.com/supplemental).

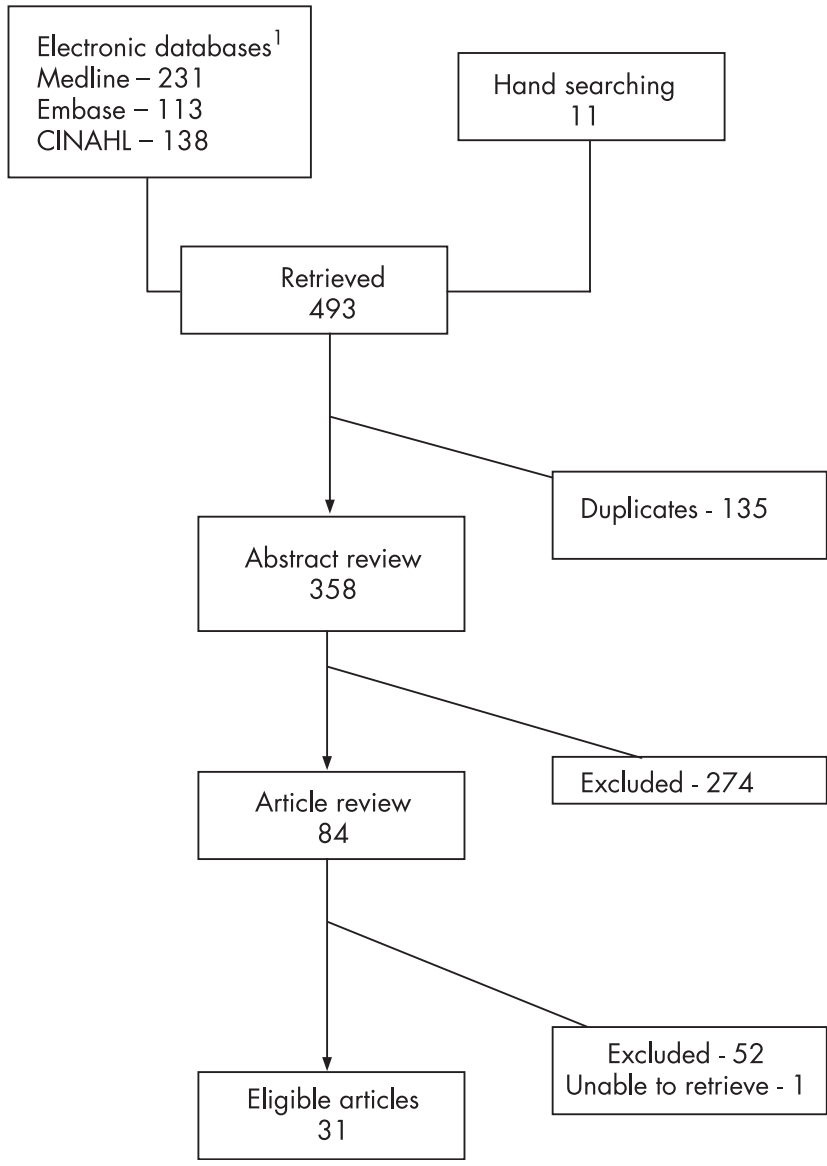

Figure 2 Summary of search and review process.

\section{Systematic literature review on medication errors Study characteristics \\ Design}

Table 1 details the characteristics of the 31 studies. Twenty-three of the 31 studies occurred within single institutions and 21 included data from $a \leqslant 1$ year period, with the minimum time period being 1 week. Twenty-two of the studies evaluated paediatric inpatients, five studies were focused on either ambulatory clinics or emergency departments and three studies evaluated the home setting. Eighteen of the studies evaluated all medications able to be dispensed in that care setting, whereas 13 studies focused on only a subset of medications (table 1).

Overall the numerator data reported was described as or was consistent with "medication errors" in 25 studies, "ADEs" in 1 study, and 5 studies reported both medication errors and ADEs. The types of medication errors reported included prescribing errors $(n=14)$, dispensing errors $(n=7)$, administering errors $(\mathrm{n}=14)$, monitoring patient for effects errors $(\mathrm{n}=1)$, MAR errors $(n=7)$, and overall lumping of all of these error types $(\mathrm{n}=14)$. There was non-uniformity in the definitions used, if they were explicitly stated, for medication errors. For example, one study defined an error as only those orders with a 10 -fold dosing error and another defined medication errors as only those with $>10 \%$ deviation from recommended dose. These differences are detailed in tables 3-8 under numerator and numerator description columns.

\section{Denominator data}

The denominator data were equally non-uniform among the studies. The possible denominators included: manual 
Table 1 Summary of article characteristics

\begin{tabular}{|c|c|c|c|c|c|c|c|}
\hline Citation & Type of study & Setting & $\begin{array}{l}\text { Type of } \\
\text { medication } \\
\text { studied }\end{array}$ & Type of numerator & Type of denominator & $\begin{array}{l}\text { How were data } \\
\text { obtained }\end{array}$ & $\begin{array}{l}\text { Types of errors } \\
\text { collected }\end{array}$ \\
\hline $\begin{array}{l}\text { Simpson } \\
\text { et } a f^{3}\end{array}$ & Interventional & $\begin{array}{l}\text { ICU patients } \\
\text { only }\end{array}$ & All types & & Patient days & $\begin{array}{l}\text { Incident/error } \\
\text { reports }\end{array}$ & All types \\
\hline $\begin{array}{l}\text { Petridou } \\
\text { et } a f^{25}\end{array}$ & $\begin{array}{l}\text { Retrospective } \\
\text { review without } \\
\text { controls }\end{array}$ & $\begin{array}{l}\text { Clinic or } \\
\text { outpatient }\end{array}$ & Vaccines & Medication errors & Time period ( 2 years) & $\begin{array}{l}\text { Incident/error } \\
\text { reports }\end{array}$ & All types \\
\hline $\begin{array}{l}\text { Cimino } \\
\text { et } a f^{8}\end{array}$ & Interventional & $\begin{array}{l}\text { ICU patients } \\
\text { only }\end{array}$ & All types & $\begin{array}{l}\text { Adverse drug events } \\
\text { and medication errors }\end{array}$ & Manual medication orders & Chart reviews & All types \\
\hline $\begin{array}{l}\text { Butte } \\
\text { et }\left.a\right|^{41}\end{array}$ & Cohort & & Immunisations & Medication errors & $\begin{array}{l}\text { Manual medication orders } \\
\text { and patients }\end{array}$ & Chart reviews & All types \\
\hline $\begin{array}{l}\text { Marino } \\
\text { et }\left.a\right|^{43}\end{array}$ & Cohort & Inpatient & All types & Medication errors & $\begin{array}{l}\text { Manual medication orders } \\
\text { and patient days }\end{array}$ & Chart reviews & All types \\
\hline $\begin{array}{l}\text { Cote } \\
\text { et }\left.a\right|^{45}\end{array}$ & & $\begin{array}{l}\text { Inpatient and } \\
\text { outpatient }\end{array}$ & $\begin{array}{l}\text { Sedatives for } \\
\text { procedures }\end{array}$ & Medication errors & Manual error reports & $\begin{array}{l}\text { Incident/error } \\
\text { reports, solicited } \\
\text { case reports }\end{array}$ & All types \\
\hline $\begin{array}{l}\text { Upperman } \\
\text { et }\left.a\right|^{47}\end{array}$ & Interventional & Inpatient & All types & Adverse drug events & Per 1000 doses dispensed & $\begin{array}{l}\text { Incident/error } \\
\text { reports }\end{array}$ & All types \\
\hline $\begin{array}{l}\text { Cowley } \\
\text { et }\left.a\right|^{48}\end{array}$ & $\begin{array}{l}\text { Incident report/ } \\
\text { case series } \\
\text { review }\end{array}$ & Inpatient & All types & Medication errors & Computerised error reports & $\begin{array}{l}\text { Incident/error } \\
\text { reports from MER } \\
\text { and MedMARx }\end{array}$ & $\begin{array}{l}\text { All types and } \\
\text { administering }\end{array}$ \\
\hline Potts et $a \beta^{\beta 0}$ & Interventional & $\begin{array}{l}\text { ICU patients } \\
\text { only }\end{array}$ & All types & $\begin{array}{l}\text { Adverse drug events } \\
\text { and medication errors }\end{array}$ & Manual medication orders & Chart reviews & $\begin{array}{l}\text { All types and } \\
\text { prescribing }\end{array}$ \\
\hline $\begin{array}{l}\text { Holdsworth } \\
\text { et } a^{\beta 5}\end{array}$ & $\begin{array}{l}\text { Retrospective } \\
\text { chart reviews } \\
\text { and staff } \\
\text { interviews }\end{array}$ & Inpatient & All types & $\begin{array}{l}\text { Preventable ADEs*as } \\
\text { determined by authors } \\
\text { and potential ADEs* }\end{array}$ & $\begin{array}{l}\text { Patient days, admissions and } \\
\text { medical record evidence of } \\
\text { ADE*: excluded errors }^{*} \\
\text { corrected before medication } \\
\text { put into MAR }\end{array}$ & $\begin{array}{l}\text { Chart reviews and } \\
\text { interviews }\end{array}$ & $\begin{array}{l}\text { All types, prescribing } \\
\text { and dispensing }\end{array}$ \\
\hline $\begin{array}{l}\text { Sangtawesin } \\
\text { et } a^{\beta 1}\end{array}$ & $\begin{array}{l}\text { Retrospective } \\
\text { review without } \\
\text { controls }\end{array}$ & Inpatient & All types & Medication errors & Admissions & $\begin{array}{l}\text { Incident/error } \\
\text { reports }\end{array}$ & $\begin{array}{l}\text { All types, prescribing, } \\
\text { dispensing and } \\
\text { administering }\end{array}$ \\
\hline Frey et $a{ }^{\beta}$ & $\begin{array}{l}\text { Incident report/ } \\
\text { case series } \\
\text { review }\end{array}$ & $\begin{array}{l}\text { ICU patients } \\
\text { only }\end{array}$ & All types & Medication errors & Manual error reports & $\begin{array}{l}\text { Incident/error } \\
\text { reports }\end{array}$ & $\begin{array}{l}\text { All types, prescribing, } \\
\text { dispensing, } \\
\text { administering and } \\
\text { MAR/documentation }\end{array}$ \\
\hline $\begin{array}{l}\text { Derrough } \\
\text { and Kitchin }\end{array}$ & $\begin{array}{l}\text { Incident report/ } \\
\text { case series } \\
\text { review }\end{array}$ & Mix & Vaccines & $\begin{array}{l}\text { Inadvertent vaccine } \\
\text { administration }\end{array}$ & $\begin{array}{l}\text { Prospective inquiries of } \\
\text { inadvertent admission of } \\
\text { vaccines: calls by providers to } \\
\text { pharmaceutical company } \\
\text { information service }\end{array}$ & $\begin{array}{l}\text { Incident/error } \\
\text { reports } \\
0\end{array}$ & Administering \\
\hline Li et $a l^{44}$ & Cross sectional & Home & $\begin{array}{l}\text { Paracetamol } \\
\text { and ibuprofen }\end{array}$ & Medication errors & $\begin{array}{l}\text { Patients } \leqslant 10 \text { receiving } \\
\text { paracetamol or ibuprofen at } \\
\text { home in past } 24 \text { hours }\end{array}$ & Survey to parents & Administering \\
\hline $\operatorname{Losek}^{46}$ & $\begin{array}{l}\text { Retrospective } \\
\text { review without } \\
\text { controls }\end{array}$ & $\begin{array}{l}\text { Emergency } \\
\text { department }\end{array}$ & $\begin{array}{l}\text { Paracetamol } \\
\text { administered } \\
\text { by emergency } \\
\text { department } \\
\text { staff }\end{array}$ & Medication errors & Patients & Chart reviews & Administering \\
\hline $\begin{array}{l}\text { Feikema } \\
\text { et }\left.a\right|^{51}\end{array}$ & Cross sectional & $\begin{array}{l}\text { Clinic or } \\
\text { outpatient }\end{array}$ & Vaccines & Extra immunisations & Patients & $\begin{array}{l}\text { Chart reviews } \\
\text { from National } \\
\text { Immunisation } \\
\text { Survey screenings }\end{array}$ & Administering \\
\hline $\begin{array}{l}\text { Goldman } \\
\text { and Scolnik }\end{array}$ & $\begin{array}{l}\text { Cross sectional } \\
\text { parental } \\
\text { interview }\end{array}$ & Home & $\begin{array}{l}\text { Paracetamol } \\
\text { administered } \\
\text { by parents at } \\
\text { home }\end{array}$ & Medication errors & Patients & Interviews & Administering \\
\hline $\begin{array}{l}\text { McErlean } \\
\text { et } a P^{53}\end{array}$ & $\begin{array}{l}\text { Cross sectional } \\
\text { parental } \\
\text { interview }\end{array}$ & Home & $\begin{array}{l}\text { Antipyretic } \\
\text { drugs } \\
\text { administered } \\
\text { by parent at } \\
\text { home }\end{array}$ & Medication errors & Patients & Interviews & Administering \\
\hline $\begin{array}{l}\text { Parshuram } \\
\text { et } a \beta^{2}\end{array}$ & $\begin{array}{l}\text { Prospective } \\
\text { observational }\end{array}$ & $\begin{array}{l}\text { ICU patients } \\
\text { only }\end{array}$ & $\begin{array}{l}\text { Morphine } \\
\text { infusion }\end{array}$ & $\begin{array}{l}\text { Discrepancies between } \\
\text { ordered and measured } \\
\text { concentrations }\end{array}$ & Number of infusions & $\begin{array}{l}\text { Liquid } \\
\text { chromatography }\end{array}$ & Dispensing \\
\hline $\begin{array}{l}\text { Cable and } \\
\text { Craft }^{26}\end{array}$ & $\begin{array}{l}\text { Retrospective } \\
\text { review without } \\
\text { controls }\end{array}$ & Inpatient & All types & $\begin{array}{l}\text { Disagreement of } \\
\text { Cardex with order }\end{array}$ & Manual medication orders & Chart reviews & MAR/documentation \\
\hline $\begin{array}{l}\text { Lehmann } \\
\text { ef } a^{P^{7}}\end{array}$ & Interventional & $\begin{array}{l}\text { ICU patients } \\
\text { only }\end{array}$ & TPN & Medication errors & $\begin{array}{l}\text { Manual medication orders } \\
\text { (TPN) }\end{array}$ & Order review & Prescribing \\
\hline
\end{tabular}


Table 1 Continued

\begin{tabular}{|c|c|c|c|c|c|c|c|}
\hline Citation & Type of study & Setting & $\begin{array}{l}\text { Type of } \\
\text { medication } \\
\text { studied }\end{array}$ & Type of numerator & Type of denominator & $\begin{array}{l}\text { How were data } \\
\text { obtained }\end{array}$ & $\begin{array}{l}\text { Types of errors } \\
\text { collected }\end{array}$ \\
\hline $\begin{array}{l}\text { Cordero } \\
\text { et } a \text { p }^{9}\end{array}$ & Interventional & $\begin{array}{l}\text { ICU patients } \\
\text { only }\end{array}$ & Gentamicin & Medication errors & $\begin{array}{l}\text { VLBW infants born } \\
\text { consecutively } 6 \text { months } \\
\text { before CPOE receiving } \\
\text { drug }\end{array}$ & $\begin{array}{l}\text { Chart reviews } \\
\text { and medical } \\
\text { records }\end{array}$ & Prescribing \\
\hline Lesar $^{36}$ & $\begin{array}{l}\text { Incident report/ } \\
\text { case series } \\
\text { review }\end{array}$ & Inpatient & All types & Medication errors & $\begin{array}{l}\text { Computerised error } \\
\text { reports, patient days and } \\
\text { admissions identified by } \\
\text { pharmacists and entered } \\
\text { into relational database }\end{array}$ & $\begin{array}{l}\text { Incident/error } \\
\text { reports }\end{array}$ & Prescribing \\
\hline $\begin{array}{l}\text { Farrar } \\
\text { et } a^{49}\end{array}$ & $\begin{array}{l}\text { Interventional } \\
\text { study }\end{array}$ & Inpatient & All types & Medication errors & $\begin{array}{l}\text { Computerised medication } \\
\text { orders }\end{array}$ & Chart reviews & Prescribing \\
\hline $\begin{array}{l}\text { Fontan } \\
\text { et } a^{\beta 4}\end{array}$ & Cohort & Inpatient & All types & Medication errors & $\begin{array}{l}\text { Manual medication orders } \\
\text { and computerised } \\
\text { medication orders }\end{array}$ & Chart reviews & $\begin{array}{l}\text { Prescribing and } \\
\text { administering }\end{array}$ \\
\hline $\begin{array}{l}\text { Kozer } \\
\text { et }\left.a\right|^{\beta 9}\end{array}$ & $\begin{array}{l}\text { Retrospective } \\
\text { review without } \\
\text { controls }\end{array}$ & $\begin{array}{l}\text { Emergency } \\
\text { department }\end{array}$ & All types & Medication errors & Manual medication orders & Chart reviews & $\begin{array}{l}\text { Prescribing and } \\
\text { administering }\end{array}$ \\
\hline $\begin{array}{l}\text { Pichon } \\
\text { et }\left.a\right|^{40}\end{array}$ & $\begin{array}{l}\text { Retrospective } \\
\text { review with } \\
\text { controls }\end{array}$ & Inpatient & All types & Medication errors & Manual medication orders & Chart reviews & $\begin{array}{l}\text { Prescribing and MAR/ } \\
\text { Documentation }\end{array}$ \\
\hline $\begin{array}{l}\text { France } \\
\text { et } a P^{4}\end{array}$ & $\begin{array}{l}\text { Incident report/ } \\
\text { case series } \\
\text { review }\end{array}$ & Inpatient & $\begin{array}{l}\text { Chemotherapy } \\
\text { only }\end{array}$ & Medication errors & Computerised error reports & $\begin{array}{l}\text { Incident/error } \\
\text { reports }\end{array}$ & $\begin{array}{l}\text { Prescribing, } \\
\text { dispensing, } \\
\text { administering and } \\
\text { MAR/documentation }\end{array}$ \\
\hline $\begin{array}{l}\text { King } \\
\text { et }\left.a\right|^{\beta 3}\end{array}$ & Interventional & Inpatient & All types & $\begin{array}{l}\text { Adverse drug events } \\
\text { and medication errors }\end{array}$ & Manual error reports & $\begin{array}{l}\text { Incident/error } \\
\text { reports }\end{array}$ & $\begin{array}{l}\text { Prescribing, } \\
\text { dispensing, } \\
\text { administering and } \\
\text { MAR/documentation }\end{array}$ \\
\hline
\end{tabular}

$A D E$, adverse drug event; CPOE, computerised physician order entry; ICU, Intensive care unit; MAR, medication administration record; MedMARx, United States Pharmacopeia database designed to reduce medication errors in hospitals; MER: medication errors reporting programme submitted to United States Pharmacopeia; TPN, total parenteral nutrition; VLBW, very low birth weight.

${ }^{*} \mathrm{ADEs}$ are defined as having the potential to produce significant injury, includes errors detected before drug administration as well as errors that did not produce significant adverse consequences, excludes errors that were identified and corrected before the medication was entered into MAR.

†ICD9, International Classification of Disease, 9th edition. Public Health Service and Health Care Financing Administration. International classification of diseases, 9th revision, clinical modification. Vols 1, 2, and 3; eighth edition. Washington, DC: Public Health Service; 1997.

¥Code 995.2: adverse effect/allergic reaction/hypersensitivity/idiosyncrasy of drug, medicinal and biological substance (due) to correct medicinal substance properly administered.

(paper-based) error reports, computerised error reports, manual medication orders, computerised medication orders, patient days, number of admissions, and time periods. Furthermore, many studies had very narrowly defined denominators, such as number of total parenteral nutrition orders or patients $<10$ years of age who received paracetamol or ibuprofen in the past 24 hours.

\section{Source of data collection}

The majority of the overall data was collected by either chart reviews $(n=14)$ or incident/error reports $(n=11)$. Although studies that used incident/error reports cannot be used to assess overall epidemiology of medication errors in children, we included them in order to provide insight into the distributional epidemiology of types of medication errors seen in children.

\section{Overall medication error results from systematic literature review}

Fourteen of 31 studies reported overall medication error data that included the entire spectrum from prescribing through to monitoring patient for effect. Table 2 gives the results of these studies. Of these, seven reported broad estimates of overall medication error rates in all children based on actual or estimated data using denominators such as patient days, admissions, or orders as opposed to evaluating only medication error reports (Simpson 2004 ${ }^{23}$; Cimino 2004 ${ }^{28}$; Potts 2004 Sangtawesin 2003 ${ }^{31}$; Holdsworth 2003 ${ }^{35}$; Kaushal 2001 $1^{42}$; Marino $2000^{43}$ ). Using the studies, the results showed a range of estimated medication errors per medication orders from $5 \%$ to $27 \%$ based on three studies with similar numerators and denominators that allow consideration together (Cimino 2004' ${ }^{28}$; Kaushal 2001 ${ }^{42}$; Marino 2000 ${ }^{43}$ ).

\section{Prescribing error results from systematic literature review on medication errors}

Fourteen studies reported medication prescribing errors (table 3). These summarised an estimated prescribing error rate per medication orders of $30 \%, 20 \%$, and $4 \%$ from the three studies that used similar numerators and denominators (Potts $2004^{30}$; Fontan 2003 ${ }^{34}$; Kaushal $2001^{42}$ ). The first two studies in this estimate appeared to have broader definitions of medication errors, which may explain the higher error rate estimates. For example, both included all types of omissions as a medication error, such as omissions of weight and prescriber's name. Three of the studies reported overall prescribing errors as rates per patient. The estimates from these studies are prescribing errors for each patient of 4-400 per 1000 patients (Sangtawesin 2003 ${ }^{31}$; Kozer 2002 ${ }^{39}$; Kaushal 2001 ${ }^{42}$ ).

\section{Dispensing error results from systematic literature review on medication errors}

Seven studies reported dispensing errors, although the design of the studies was very different (table 4). Looking at the greatest commonality between these studies-namely, those studies based on error reports, the estimates of the percentage of reported errors that are related to dispensing vary widely from 5\% to 58\% (France 2004 ${ }^{24}$; King 2003 $3^{33}$; Frey 2002 $2^{38}$ ). 
Table 2 Summary of studies with overall medication error results

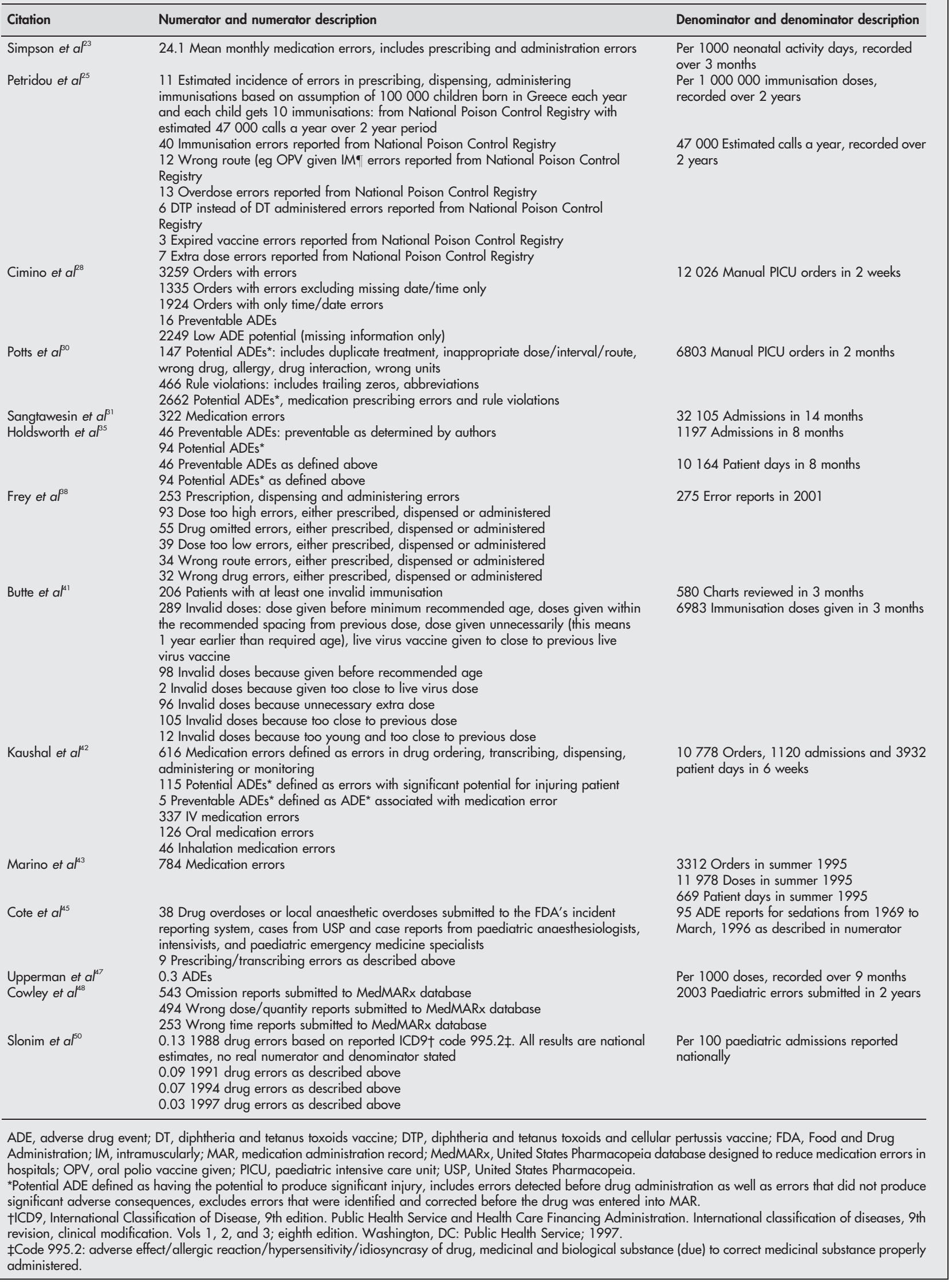


Table 3 Prescribing error results summary

\begin{tabular}{|c|c|c|}
\hline Citation & Numerator and numerator description & Denominator and denominator description \\
\hline France et $a^{24}$ & 71 Chemotherapy ordering errors: includes dosing, omission and wrong date errors & $\begin{array}{l}97 \text { Electronically reported chemotherapy errors in } \\
13 \text { months }\end{array}$ \\
\hline Lehmann et $a^{P^{7}}$ & $\begin{array}{l}60 \text { TPN errors that required pharmacist to contact provider: includes osmolality } \\
\text { problems, insufficient fluid, calculation errors, omissions }\end{array}$ & 557 TPN orders in 1.5 months \\
\hline Cordero et al ${ }^{2}$ & $\begin{array}{l}14 \text { Gentamicin prescription dosage errors: prescribed dose }>10 \% \text { deviation from } \\
\text { recommended dose } \\
5 \text { Gentamicin overdoses: }>10 \% \text { overdose } \\
9 \text { Gentamicin underdoses: }>10 \% \text { underdose }\end{array}$ & $\begin{array}{l}105 \text { VLBW infants born consecutively } 6 \text { months } \\
\text { before CPOE receiving drug }\end{array}$ \\
\hline Potts et $a^{\beta 0}$ & 2049 Medication prescribing errors: includes weight not available, missing information & 6803 Manual PICU orders in 2 months \\
\hline Sangtawesin et $a^{\beta 1}$ & 114 Prescribing errors: includes wrong dose, wrong choice, known allergy and others & 32105 Admissions in 14 months \\
\hline King et $a^{\beta 3}$ & 13 Prescribing medication errors & 416 Medication errors in 3 years \\
\hline \multirow[t]{7}{*}{ Fontan et $a^{\beta 4}$} & $\begin{array}{l}937 \text { Prescribing errors: includes any error in the prescription of drug's name, form, } \\
\text { dosage, route, any omission of these prescribing items including prescriber's name } \\
\text { and any drug interaction. }\end{array}$ & 4532 Prescribed drugs in 2 months \\
\hline & $\begin{array}{l}419 \text { Computerised prescribing errors as defined above } \\
518 \text { Hand written prescribing errors } \\
44 \text { Wrong form errors }\end{array}$ & $\begin{array}{l}3943 \text { Computerised prescribed drugs in } 2 \text { months } \\
589 \text { Hand written prescribed drugs in } 2 \text { months } \\
4532 \text { Prescribed drugs in } 2 \text { months }\end{array}$ \\
\hline & 19 Wrong route errors & \\
\hline & 47 Wrong dosage errors & \\
\hline & 34 Wrong dosage form errors & \\
\hline & 587 Omission errors & \\
\hline & 152 "Caution" drug interactions & \\
\hline \multirow[t]{2}{*}{ Holdsworth et $a^{\beta 5}$} & $\begin{array}{l}0 \text { "Contraindicated" and "not advised" drug interactions } \\
35 \text { Preventable ADEs that were underdose, wrong dose and overdose: preventable was } \\
\text { determined by authors }\end{array}$ & 46 Preventable ADEs in 8 months \\
\hline & 39 Potential ADEs* that were underdose and overdose & 94 Potential ADEs* in 8 months as defined above \\
\hline Lesar $^{36}$ & $\begin{array}{l}39 \text { Prescribing errors occurring in paediatric patients: all errors were prevented before } \\
\text { reaching patient } \\
0.5310 \text {-Fold error rate in paediatric patients } \\
0.9810 \text {-Fold error rate in paediatric patients }\end{array}$ & $\begin{array}{l}200 \text { Error reports identified by pharmacists and } \\
\text { entered into a relational database in } 6 \text { months } \\
\text { Per } 100 \text { admissions, recorded over } 6 \text { months } \\
\text { Per } 1000 \text { patient days, recorded over } 6 \text { months }\end{array}$ \\
\hline Frey et $\left.a\right|^{\beta 8}$ & $\begin{array}{l}102 \text { Overall prescribing errors } \\
9 \text { Illegible errors } \\
37 \text { Calculation errors } \\
22 \text { Wrong unit errors (eg } \mathrm{ml} \text { instead of } \mathrm{mg} \text { ) }\end{array}$ & 275 Error reports in 2001 \\
\hline Kozer et $a{ }^{\beta 9}$ & $\begin{array}{l}154 \text { Prescribing errors } \\
117 \text { Wrong frequency errors } \\
133 \text { Wrong dose errors } \\
5 \text { Wrong drug errors } \\
7 \text { Wrong route errors }\end{array}$ & $\begin{array}{l}1532 \text { Charts reviewed in } 12 \text { randomly selected } \\
\text { days in summer }\end{array}$ \\
\hline Pichon et $a f^{40}$ & $\begin{array}{l}76 \text { Incomplete non-chemotherapy orders defined as omissions: number of doses } \\
\text { missing, route missing, dose missing } \\
89 \text { Non-chemotherapy order omissions: more than one omission possible in a single } \\
\text { order }\end{array}$ & 198 Non-chemotherapy orders \\
\hline & 19 Omissions on non-chemotherapy PRN¥ orders & 22 Non-chemotherapy PRN $¥$ orders \\
\hline Kaushal et $a t^{42}$ & $\begin{array}{l}454 \text { Physician ordering medication errors } \\
91 \text { Physician ordering potential ADEs*: defined as errors with significant potential for } \\
\text { injuring patient }\end{array}$ & $\begin{array}{l}10778 \text { Orders, } 1120 \text { admissions and } 3932 \\
\text { patient days in } 6 \text { weeks }\end{array}$ \\
\hline Farrar et a ${ }^{49}$ & $\begin{array}{l}29 \text { Prescribing errors for non-paediatricians for orders reviewed } \\
17 \text { Prescribing errors for paediatricians for orders reviewed }\end{array}$ & $\begin{array}{l}38 \text { Non-paediatricians' orders reviewed } \\
65 \text { Paediatricians' orders reviewed }\end{array}$ \\
\hline $\begin{array}{l}\text { ADE, adverse druc } \\
\text { administered as re } \\
\text { *Potential } A D E \text { def } \\
\text { significant adverse }\end{array}$ & $\begin{array}{l}\text { nt; } C P O E \text {, computerised physician order entry; MAR, medication administration recor } \\
\text { ed; TPN, total parenteral nutrition; VLBW, very low birth weight. } \\
\text { as having the potential to produce significant injury, includes errors detected before } d \\
\text { sequences, excludes errors that were identified and corrected before the drug was en }\end{array}$ & $\begin{array}{l}\text { U, paediatric intensive care unit; PRN, drug } \\
\text { Iministration as well as errors that did not produce }\end{array}$ \\
\hline
\end{tabular}

Table 4 Dispensing error results summary

\begin{tabular}{|c|c|c|}
\hline Citation & Numerator and numerator description & Denominator and denominator description \\
\hline $\begin{array}{l}\text { France et } a l^{4} \\
\text { Sangtawesin et } a l^{\beta 1} \\
\text { Parshuram et } a l^{\beta 2}\end{array}$ & $\begin{array}{l}9 \text { Preparation chemotherapy errors } \\
112 \text { Dispensing errors } \\
150 \text { Discrepancies of }>10 \% \text { between ordered and } \\
\text { measured infusions of morphine } \\
13 \text { Twofold or greater discrepancy between ordered } \\
\text { and measured infusions of morphine }\end{array}$ & $\begin{array}{l}97 \text { Electronically reported chemotherapy errors in } 13 \text { months } \\
32105 \text { Admissions in } 14 \text { months } \\
232 \text { Infusions in } 7 \text { months }\end{array}$ \\
\hline $\begin{array}{l}\text { King et } a^{\beta 3} \\
\text { Holdsworth et } a^{\beta 5} \\
\text { Frey et }\left.a\right|^{\beta 8} \\
\text { Kaushal et } a^{\mu 2}\end{array}$ & $\begin{array}{l}19 \text { Dispensing errors } \\
39 \text { Number of dispensing potential ADEs* } \\
162 \text { Dispensing errors } \\
6 \text { Pharmacy dispensing medication errors } \\
4 \text { Pharmacy dispensing potential ADEs*: defined as } \\
\text { errors with significant potential for injuring patient }\end{array}$ & $\begin{array}{l}416 \text { Medication errors in } 3 \text { years } \\
94 \text { Potential ADEs* in } 8 \text { months } \\
275 \text { Error reports in } 2001 \\
10778 \text { Orders, or } 1120 \text { admissions, or } 3932 \text { patient days in } 6 \text { weeks }\end{array}$ \\
\hline
\end{tabular}

$A D E$, adverse drug event; $M A R$, medication administration record.

*Potential ADE defined as having the potential to produce significant injury, includes errors detected before drug administration as well as errors that did not produce significant adverse consequences, excludes errors that were identified and corrected before the drug was entered into MAR. 
Table 5 Administering error results summary

\begin{tabular}{|c|c|c|}
\hline Citation & Numerator and numerator description & Denominator and denominator description \\
\hline France et $a^{24}$ & 13 Administering chemotherapy errors & $\begin{array}{l}97 \text { Electronically reported chemotherapy errors in } \\
13 \text { months }\end{array}$ \\
\hline Sangtawesin et $a^{\beta 1}$ & $\begin{array}{l}49 \text { Administering errors: includes wrong time, omission error, wrong strength, } \\
\text { unauthorised drug, wrong patient, extra dose, wrong route, wrong dosage form }\end{array}$ & 32105 Admissions in 14 months \\
\hline King et $a^{\beta 3}$ & 314 Administering errors & 416 Medication errors in 3 years \\
\hline Fontan et $a^{\beta 4}$ & $\begin{array}{l}1077 \text { Administering errors defined as any deviation between prescribed and } \\
\text { administered drugs: includes extra/omitted dose, wrong route, wrong time and } \\
\text { patient non-compliant } \\
57 \text { Extra dose errors } \\
454 \text { Dose omission errors } \\
17 \text { Wrong dose errors } \\
2 \text { Wrong route errors } \\
8 \text { Patient non-compliant errors } \\
539 \text { Wrong time errors }\end{array}$ & $\begin{array}{l}4589 \text { Opportunities for administering errors: the } \\
\text { sum of administered drugs and omitted drugs in } \\
2 \text { months }\end{array}$ \\
\hline $\begin{array}{l}\text { Derrough and } \\
\text { Kitchin }^{37}\end{array}$ & $\begin{array}{l}161 \text { Inadvertent administration of vaccine to children: includes out of schedule } \\
\text { according to the national recommendations, error in reconstitution of vaccine or } \\
\text { diluent used, vaccine given at inappropriate age, inappropriate interval between } \\
\text { vaccines, wrong vaccine (eg DTP for DT), expired vaccine, vaccine contraindicated. } \\
\text { From pharmaceutical company telephone-based vaccine information service }\end{array}$ & $\begin{array}{l}302 \text { Inadvertent vaccine administrations (all age } \\
\text { groups) in } 1 \text { year }\end{array}$ \\
\hline Frey et $a^{\beta 8}$ & 200 Administering errors & 275 Error reports in 2001 \\
\hline Kozer et a ${ }^{\beta 9}$ & 59 Administering errors & $\begin{array}{l}1532 \text { Charts reviewed in } 12 \text { randomly selected days } \\
\text { in summer }\end{array}$ \\
\hline Kaushal et $a t^{42}$ & $\begin{array}{l}78 \text { Nurse administering medication errors } \\
5 \text { Nurse administering potential ADEs*: defined as errors with significant potential } \\
\text { for injuring patient }\end{array}$ & $\begin{array}{l}10788 \text { Orders, } 1120 \text { admissions and } 3932 \text { patien } \\
\text { days in } 6 \text { weeks }\end{array}$ \\
\hline \multirow[t]{2}{*}{ Li et $\left.a\right|^{44}$} & $\begin{array}{l}87 \text { Incorrect paracetamol doses at home } \\
66 \text { Paracetamol underdoses at home } \\
21 \text { Paracetamol overdoses at home } \\
6 \text { Paracetamol doses given more frequently than } 4 \text { hours at home }\end{array}$ & $\begin{array}{l}140 \text { Patients who received home administrations of } \\
\text { paracetamol in past } 24 \text { hours, recorded over } \\
3 \text { months }\end{array}$ \\
\hline & $\begin{array}{l}19 \text { Incorrect ibuprofen doses at home } \\
9 \text { Ibuprofen underdoses at home } \\
10 \text { Ibuprofen overdoses at home } \\
28 \text { Ibuprofen doses given more frequently than } 6 \text { hours at home }\end{array}$ & $\begin{array}{l}74 \text { Patients who received home administrations of } \\
\text { ibuprofen in past } 24 \text { hours, recorded over } 3 \text { month }\end{array}$ \\
\hline $\operatorname{Losek}^{46}$ & 34 Paracetamol doses outside standing orders of $10-15 \mathrm{mg} / \mathrm{kg}$ & $\begin{array}{l}156 \text { Emergency department patients receiving } \\
\text { paracetamol in } 1 \text { week }\end{array}$ \\
\hline Cowley et a ${ }^{48}$ & 1007 Administering errors submitted to MedMARx database & $\begin{array}{l}1956 \text { Paediatric errors with phase of error indicatec } \\
\text { submitted to MedMARx in } 2 \text { years }\end{array}$ \\
\hline McErlean et $a^{p^{3}}$ & 53 Incorrect doses of antipyretic drug at home compared with recommended dose & $\begin{array}{l}118 \text { Patients who received home administration of } \\
\text { antipyretic drugs }\end{array}$ \\
\hline
\end{tabular}

Administration error results from systematic literature review on medication errors

Fourteen studies reported administration errors for children (table 5). Six of these studies were medication-specific with two focused on vaccines and four focused on paracetamol and/ or ibuprofen only. Of the three studies which were global in scope, nevertheless, variation among the studies in numerator and denominator definitions and methods of data collection made comparisons difficult. Using the one study that defined "total opportunities for administering errors" as the global denominator, the distributional epidemiology of administration errors shows that the majority of these errors involved either dose omissions (42\%) or wrong time of administration (50\%) (Fontan 2003 ${ }^{34}$ ).

Table 6 MAR documentation error results summary

\begin{tabular}{|c|c|c|}
\hline Citation & Numerator and numerator description & Denominator and denominator description \\
\hline France et $a^{24}$ & 3 Chemotherapy transcription errors & $\begin{array}{l}97 \text { Electronically reported chemotherapy errors in } \\
13 \text { months }\end{array}$ \\
\hline Cable and $\mathrm{Craft}^{26}$ & $\begin{array}{l}109 \text { Disagreement with Cardex } \\
49 \text { Major causes of disagreement: different dose, wrong medication, wrong } \\
\text { frequency or duration, missing route } \\
39 \text { Orders not on Cardex }\end{array}$ & $\begin{array}{l}540 \text { Randomly selected paediatric medication orders } \\
\text { drawn from over } 2 \text { years }\end{array}$ \\
\hline Sangtawesin et $a^{\beta 1}$ & 46 "Order processing" errors & 32105 Admissions in 14 months \\
\hline King et $\left.a\right|^{\beta 3}$ & 70 Transcription errors & 416 Reported medication errors in 3 years \\
\hline Frey et $a^{\beta 8}$ & 58 Errors in transcription of physicians order onto medication chart & 275 Error reports in 2001 \\
\hline Pichon et $\left.a\right|^{40}$ & $\begin{array}{l}41 \text { Non-chemotherapy transcription errors } \\
16 \text { Chemotherapy transcription errors }\end{array}$ & $\begin{array}{l}198 \text { Non-chemotherapy orders } \\
135 \text { Chemotherapy orders }\end{array}$ \\
\hline Kaushal et $a^{\mu 2}$ & $\begin{array}{l}85 \text { Documentation medication errors } \\
9 \text { Documentation potential ADEs: defined as errors with significant potential } \\
\text { for injuring patient }\end{array}$ & $\begin{array}{l}10778 \text { Orders, or } 1120 \text { admissions, or } 3932 \text { patient } \\
\text { days in } 6 \text { weeks }\end{array}$ \\
\hline
\end{tabular}


Table 7 Monitoring for effect error results summary

\begin{tabular}{lll}
\hline Citation & Numerator and numerator description & Denominator and denominator description \\
\hline Kaushal et a ${ }^{42}$ & $\begin{array}{l}4 \text { Monitoring medication errors; 0 Monitoring potential ADEs } \\
\text { defined as errors with significant potential for injuring patient }\end{array}$ & $\begin{array}{l}10 \text { 778 Orders, or } 1120 \text { admissions, or 3932 patient days } \\
\text { in } 6 \text { weeks }\end{array}$ \\
\hline ADE, adverse drug event. &
\end{tabular}

$M A R /$ documentation error results from systematic literature review on medication errors

Seven studies evaluated documentation errors among children (table 6). The estimate of transcription errors from these studies varies from $<1 \%$ of orders to $20 \%$ of orders having a transcription error.

\section{Monitoring the patient for effect error results from} systematic literature review on medication errors Only one study, listed in table 7, reported monitoring a patient for effect errors and estimated, via chart review, that the incidence was four errors per 1000 patients (Kaushal 2001 ${ }^{42}$ ).

\section{Distributional epidemiology of medication error from error reports}

Four studies provided data that can be synthesised to understand the distributional epidemiology of medication errors in paediatrics based on error reports (France 2004 ${ }^{24}$; King 2003 ${ }^{33}$; Lesar 2002 ${ }^{36}$; Frey $2002^{38}$ ). Such syntheses are difficult because each study location undoubtedly has different safety culture climates. The safety culture will clearly influence who completes error reports, how often they complete error reports, and what types of event are reported. Little is known about how bias in reporting influences the distributional epidemiology of medication errors. Two of these studies provided data on all medications relative to prescribing, dispensing, administering, and documentation errors (King 2003; $;^{33}$ Frey $2002^{38}$ ). Between these two studies, the distributional epidemiological estimates of the relative percentages of error types are: prescribing 3-37\%, dispensing $5-58 \%$, administering $72-75 \%$, and documentation $17-21 \%$.

\section{Estimates of the severity of medication errors for patients}

Only 11 studies categorised medication errors by severity of outcome for the patient (Simpson 2004 ${ }^{23}$; France 2004 ${ }^{24}$; Cimino

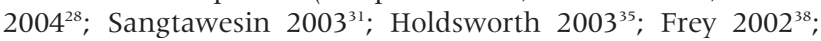
Kozer 2002 ${ }^{39}$; Kaushal 2001 ${ }^{42}$; Marino 2000 ${ }^{43}$; Upperman 2005 ${ }^{47}$; Cowley $2001^{48}$ ). Among these studies, however, at least four different scales were used to rank error severity from scales with two categories to scales with nine categories.

\section{Synthesis of recommendations to reduce medication errors for children}

We identified a total of 26 unique recommendations for strategies to reduce medication errors from national entities. The recommendations ranged from equipment/software tools, representation of personnel on groups making decisions on paediatric medications, training and competency of personnel, policies, clear labelling, continuous quality improvement efforts, clear and accurate documentation, standardisation, patient education, and teamwork improvement. Table 8 summarises these recommendations and the paediatric specific evidence behind. In short, none of these recommendations was based on published evidence of effectiveness in children. The vast majority of recommendations were based on expert opinion $(n=22)$, with the remainder being based on studies in adult populations $(\mathrm{n}=4)$. No recommendation had support- ing paediatric specific evidence on efficacy, cost effectiveness, feasibility, appropriateness in different settings, and institutional barriers or risks.

\section{CONCLUSIONS}

Since the Institute of Medicine To Err Is Human report ${ }^{1}$ a significant amount of research has been done on medication errors in children, and a significant number of recommendations have been made by various entities on how to make medication administration safer for children. There can be no doubt, based on this evidence, that medication errors are a significant percentage of medical errors in children. Our review estimates that $5-27 \%$ of medication orders for children contain an error somewhere along the spectrum of the entire delivery process involving prescribing, dispensing, and administering based on three studies (Cimino 2004' $2000^{43}$ ). Our review also estimates that there are $100-400$ prescribing errors per 1000 patients and highlights that the majority of research to date has focused on the prescribing step of medication delivery (Kozer 2002 ${ }^{39}$; Kaushal 2001 ${ }^{42}$ ).

Looking at error reporting systems, it is clear that each step of the medication process is error prone, although the majority of research has focused on prescribing errors. Our evidence based estimates at the overall "share of the pie" that each step contributes to the overall rate of medication errors among children are the following: prescribing 3-37\%, dispensing 5$58 \%$, administering $72-75 \%$, and documentation $17-21 \%$ (King $2003^{33}$; Frey $2002^{38}$ ).

Overall, our depth of understanding of the epidemiology of paediatric medication errors remains poor. Our systematic literature review on medication errors highlights the fact that estimates of the incidence of medication errors in children are severely hampered by the lack of uniform definitions of medication errors (numerator data) and study population (denominator) among studies and by the different means of data collection used to identify errors.

Also of importance, many studies did not explicitly define medication errors. A recently published report looking only at prescribing errors in children highlighted the great difficulty in defining what a medication error is. ${ }^{54}$ Barriers to defining medication errors in children and to then being able to measure the epidemiology of medication errors include: off-label use of medications with dosage ranges extrapolated from adult literature, different recommendations for dosing ranges for the same medication from different sources, and unclear rules as to when adult doses may be appropriate for children. None of the studies looking at all medications with details on prescribing errors stated what the "correct" dosing range was that guided their definitions and data collection.

Focusing on the source of data, the vast majority of studies evaluated in this report relied on either chart review or error reports, with a handful using administrative or registry data. Although each mode of identifying medication errors has strengths and weaknesses and will produce varying results, it seems likely that an ideal error identification system may involve multiple data sources and potentially include triangulation between administrative data, chart review, and voluntary error reports of critical incidents in order to maximise the 
Table 8 Approaches recommended to reduce medication errors in paediatric

\begin{tabular}{|c|c|c|c|c|}
\hline Approaches to reduce medication errors & $\begin{array}{l}\text { Entities supporting } \\
\text { approach (reference } \\
\text { citations at end of table) }\end{array}$ & $\begin{array}{l}\text { Based on published } \\
\text { effectiveness evidence } \\
\text { specific for children? }\end{array}$ & $\begin{array}{l}\text { Alternative } \\
\text { processes used } \\
\text { to support approach }\end{array}$ & $\begin{array}{l}\text { Efficacy, cost, feasibility, } \\
\text { appropriateness in different } \\
\text { settings, barriers data } \\
\text { available for children? }\end{array}$ \\
\hline \multirow{7}{*}{ 1. Computerised provider order entry } & $A H R Q$ report & No & $\begin{array}{l}\text { Adult data } \\
\text { A }\end{array}$ & No \\
\hline & $\mathrm{AAP} / \mathrm{NICHQ}$ & No & Expert opinion & No \\
\hline & IOM report & No & Adult data & No \\
\hline & NQF & No & Adult data & No \\
\hline & MHA & No & Adult data & No \\
\hline & NCC MERP & No & Expert opinion & No \\
\hline & AAP & No & Expert opinion & No \\
\hline \multirow{2}{*}{ 2. Automated dispensing devices } & ISMP, PPAG, AHA & No & Expert opinion & No \\
\hline & $A H R Q$ report & No & Adult data & No \\
\hline \multirow[t]{2}{*}{ 3. Paediatric presence with formulary management } & ISMP, PPAG, AHA & No & Expert opinion & No \\
\hline & AAP & No & Expert opinion & No \\
\hline \multirow{4}{*}{ 4. Appropriate and competent pharmacy personnel and environment } & ISMP, PPAG, AHA & No & Expert opinion & No \\
\hline & NQF & No & Expert opinion & No \\
\hline & NCC MERP & No & Expert opinion & No \\
\hline & AAP & No & Expert opinion & No \\
\hline \multirow[t]{2}{*}{ 5. Pharmacist available "on call" when pharmacy is closed } & ISMP, PPAG & No & Expert opinion & No \\
\hline & MHA & No & Expert opinion & No \\
\hline \multirow[t]{5}{*}{ 6. Policies on verbal orders } & ISMP, PPAG, AHA & No & Expert opinion & No \\
\hline & JCAHO & No & Expert opinion & No \\
\hline & NQF & No & Expert opinion & No \\
\hline & NCC MERP & No & Expert opinion & No \\
\hline & AAP & No & Expert opinion & No \\
\hline 7. Clear and accurate labelling of medications & ISMP, PPAG, AHA & No & Expert opinion & No \\
\hline & NQF & No & Expert opinion & No \\
\hline & NCC MERP & No & Expert opinion & No \\
\hline 8. Quality improvement efforts with drug use evaluation and medication & ISMP, PPAG, AHA & No & Expert opinion & No \\
\hline error reporting and review & MHA & No & Expert opinion & No \\
\hline & NCC MERP & No & Expert opinion & No \\
\hline & AAP & No & Expert opinion & No \\
\hline 9. Healthcare workers have access to current clinical information and & ISMP, PPAG, AHA & No & Expert opinion & No \\
\hline references & IOM report & No & Expert opinion & No \\
\hline & MHA & No & Expert opinion & No \\
\hline & NCC MERP & No & Expert opinion & No \\
\hline & AAP & No & Expert opinion & No \\
\hline 10. Emergency medication dosage calculation tools & ISMP, PPAG & No & Expert opinion & No \\
\hline 11. Accurate documentation of medication administration & ISMP, PPAG & No & Expert opinion & No \\
\hline & MHA & No & Expert opinion & No \\
\hline & IOM report & No & Expert opinion & No \\
\hline & JCAHO & No & Expert opinion & No \\
\hline & NCC MERP & No & Expert opinion & No \\
\hline 13. Training of all healthcare providers in appropriate medication & ISMP, PPAG & No & Expert opinion & No \\
\hline prescribing, labelling, dispensing, monitoring, and administration & IOM report & No & Expert opinion & No \\
\hline & JCAHO & No & Expert opinion & No \\
\hline & NQF & No & Expert opinion & No \\
\hline & MHA & No & Expert opinion & No \\
\hline & NCC MERP & No & Expert opinion & No \\
\hline & AAP & No & Expert opinion & No \\
\hline 14. Patient education on drugs & ISMP, AHA & No & Expert opinion & No \\
\hline & IOM report & No & Expert opinion & No \\
\hline & MHA & No & Expert opinion & No \\
\hline & NCC MERP & No & Expert opinion & No \\
\hline & AAP & No & Expert opinion & No \\
\hline 15. Direct participation of pharmacists in clinical care & AHRQ report & No & Some studies & No \\
\hline & IOM report & No & Expert opinion & No \\
\hline & NQF & No & Expert opinion & No \\
\hline 16. Computer detection/alert systems for adverse drug events & AHRQ report & No & Some studies & No \\
\hline 17. Reducing adverse drug events related to anticoagulants & $A H R Q$ report & No & Some studies & No \\
\hline 18. Unit dose drug distribution systems & $A H R Q$ report & No & Some studies & No \\
\hline & AHA & No & Expert opinion & No \\
\hline & NQF & No & Expert opinion & No \\
\hline & MHA & No & Expert opinion & No \\
\hline 19. Special procedures and written protocols for high alert drugs & AHA & No & Expert opinion & No \\
\hline & IOM report & No & Expert opinion & No \\
\hline & NQF & No & Expert opinion & No \\
\hline & JCAHO & No & Expert opinion & No \\
\hline & MHA & No & Expert opinion & No \\
\hline 20. Use pharmaceutical software & IOM report & No & Expert opinion & No \\
\hline & MHA & No & Expert opinion & No \\
\hline 21. Pharmacy-based IV admixture systems & MHA & No & Expert opinion & No \\
\hline 22. Use of bar coding for medication administration & MHA & No & Expert opinion & No \\
\hline & NCC MERP & No & Expert opinion & No \\
\hline 23. Standardise equipment (e.g., pumps, weight scales) & AAP & No & Expert opinion & No \\
\hline 24. Standardise measurement systems (kilograms) & AAP & No & Expert opinion & No \\
\hline 25. Standardise order sheets to include areas for weight and allergies & AAP & No & Expert opinion & No \\
\hline $\begin{array}{l}\text { 26. Encourage team environment for review of orders among nurses, } \\
\text { pharmacists, prescribers }\end{array}$ & AAP & No & Expert opinion & No \\
\hline $\begin{array}{l}\text { PPAG, Pediatric Pharmacy Advocacy Group }{ }^{4-6} \text {; ISMP, Institute for Safe M } \\
\text { Initiative for Children's Healthcare Quality }{ }^{9-1} ; \text { IOM, Institute of Medicine'; } \\
\text { of Medical Errors }{ }^{15}{ }^{16} \text {; NCC MERP, National Coordinating Council for Me } \\
\text { Commission on Accreditation of Healthcare Organizations. }{ }^{2122}\end{array}$ & $\begin{array}{l}\text { lication Practices } \\
\text { IQf } ; \text { AHA National Quality For } \\
\text { ication errors Reporting }\end{array}$ & $\begin{array}{l}\text {; } M \text { MHA, Mas } \\
\text { vention }^{17}{ }^{18} \text {; }\end{array}$ & $\begin{array}{l}\text { 8; } \mathrm{AAP} / \mathrm{NICHQ}, \mathrm{Am} \\
\text { sital Association } / \mathrm{M} \\
\text { cy for Healthcare Re }\end{array}$ & $\begin{array}{l}\text { Academy of Pediatrics/Nationo } \\
\text { usetts Coalition for the Preventior } \\
\text { and Quality }{ }^{19} \text {; JCAHO, Joint }\end{array}$ \\
\hline
\end{tabular}


ability to identify events at each step of the process. Taken alone, each data source has significant limitations for defining the epidemiology of medication errors. Administrative data, as analysed here, are inexpensive, nearly universal, and permit unsolicited identification of potential events, although the depth of clinical information is limited. Chart review, on the other hand, provides in-depth clinical information but is fairly expensive to implement on a large scale and is limited by what is documented in the chart. Lastly, voluntary critical incident reporting depends completely on the compliance of providers with reporting but does provide real time in-depth clinical insights. Interdigitated use of these types of data collection would create a system less likely to produce a biased estimate of the epidemiology.

These significant limitations of the examined studiesnamely, differing definitions of the numerators and denominators, lack of consistent definition of medication error, less robust and/or narrowly focused methodologies, and the aforementioned short time frames of data collection and single institutional experiences in these studies, make it very difficult, if not impossible, to generalise easily the findings to all healthcare settings. Indeed the vast ranges on some of the results for estimates of different types of medication errors bear testament to this difficulty.

Despite the limitations of the available literature, several key findings warrant discussion and suggest a further national agenda for medication errors in children.

First, standardisation of recommended doses for children is an essential step to enable providers, researchers, and developers of technological solutions for prescribing to speak a common and uniform language on what doses are acceptable and what doses are in error for children. For example, a recent review exploring the limitations of recommended doses for children found a nearly twofold difference in recommended doses of oxycodone, a narcotic, among three widely used references while a fourth reference simply listed no weightbased dose recommendation. ${ }^{54}$ In a recently published study on paediatric ambulatory medication errors, one key finding was that no fewer error rates occurred at the one of three sites evaluated that used an electronic prescription writer. ${ }^{55}$ This last finding was probably due to the absence of paediatric-specific dosing logic in the electronic prescription writer. Despite the push for computerised order entry and prescribing, the lack of uniform agreement on standard paediatric doses is at least part of the reason for the usual absence of paediatric-specific dosing tables powering most commercially available computerised order entry tools. Without standard paediatric doses, and requirements that these dosage rules are built into computerised prescribing tools, children will fail to reap the benefit of information technology in the medication delivery process.

Second, standardisation of definitions of medication errors is a clear need at hand. As examples of this problem based on the studies examined here, the range of definitions of medication errors included medications prescribed at $>10 \%$ of the recommended dose all the way up to medications prescribed at 10 -fold the recommended dose. The vast majority of the articles simply did not describe the details of the definition of a medication error that was used. Comparably, looking at the entire medication delivery system, some articles did not include errors that were detected before they reached the patient, whereas other articles counted these events as errors. This ambiguity about what exactly is a medication error also permits a wide range of severity of errors to be lumped together. For example, some of the studies counted as medication errors orders that were lacking a prescriber's signature. Although this is clearly an error, the magnitude of potential harm to patients is substantially different from that of orders with dosage errors.
Without standardised guidance, all these vastly different interpretations of medication errors are lumped together and make elucidation of high priority areas difficult.

Third, despite much work on medication errors in the inpatient setting, our review identified only a handful of research on medication errors in the emergency department, ambulatory care, and home environments. All of these are critical targets for future research.

Fourth, most of the research to date has been skewed on prescribing errors. Our review of error reporting systems' data clearly shows that the medication process steps of dispensing and administering are as error-prone, if not more so, than prescribing. Understanding the unique risks for children in these two steps is critical in order to understand better which interventions will remedy the risks. Unlike the medication process for adults, these steps for children rely much more heavily on manual compounding of liquid medications and administration to patients who are unable to perform their own medication safety checks. These facts may well make the dispensing and administering of medications more error prone for children than adult patients.

Last, our synthesis of the various medication error reduction strategies recommended by national bodies resoundingly illustrated the lack of paediatric-specific evidence. However, it is inarguable that many items on the list of reduction strategies do not need multiple clinical trials to prove their impact. High cost or high resource problems such as computerised order entry, automated dispensing devices, and use of bar coding for medication administration clearly do need high quality evidence in order to foster use and, perhaps more importantly, need to have paediatric-specific evidence. Many other items, on the other hand, are relatively inexpensive, and many even broach on commonsense based on knowledge of human factors. Strategies in this latter category include: paediatric presence on Formulary committees, appropriate and competent pharmacy personnel and environment, policies on verbal orders, and clear and accurate medication labelling. The lack of paediatric-specific data on these types of recommendations is non-troubling. Perhaps more troubling is the enormous scope of recommendations coming from numerous official bodies. Such a piecemeal recommendation path leaves most providers unclear about which of the recommendations has a greater priority should they be faced with human or monetary resource limitations. National research and efforts to summarise and endorse recommendations could help care givers prioritise safety efforts and ensure that the most promising strategies of those recommended are broadly implemented first.

In summary, our review of the literature on medication errors in children and on medication error reduction strategies highlights without question that we know medication errors occur across the entire spectrum of prescribing, dispensing and administering and are a significant source of concern for paediatric patients. Furthermore, the research also confirms that medication errors are a significant concern across all settings of care, including within the home. There can be no doubt of the need for greater understanding of all the aspects of medication errors discussed here so that effective interventions and policy can be crafted. This desired understanding, however, needs a firm foundation of standardisation for issues such as dose ranges, definitions of medication errors, and even for prioritisation of implementation of medication error reduction strategies.

\section{ACKNOWLEDGEMENTS}

This research was supported by contract M-150-IOM-2005-001 from the Institute of Medicine. The Institute of Medicine helped design the study and has given approval for publication. 


\section{Authors' affiliations}

Marlene R Miller, Michael L Rinke, Department of Pediatrics, Johns Hopkins University, Baltimore, Maryland, USA

Karen A Robinson, Division of Internal Medicine, Department of Medicine, Johns Hopkins University, Baltimore, Maryland, USA

Lisa H Lubomski, Peter J Pronovost, Department of Anesthesia and Critical Care Medicine, Johns Hopkins University, Baltimore, Maryland, USA

\section{REFERENCES}

Institute of Medicine. To err is human: building a safer health system Washington, DC: National Academy Press, 1999

2 Kaushal $\mathbf{R}$, Jaggi $\mathrm{T}$, Walsh $\mathrm{K}$, et al. Pediatric medication errors: what do we know? What gaps remain? Ambul Pediatr 2004:4:73-81.

3 Kaushal R, Bates DW, Landrigan C, et al. Medication errors and adverse drug events in pediatric inpatients. JAMA 2001;285:2114-20.

4 Institute for Safe Medication Practices. http://www.ismp.org/pressroom/ PR20020606.pdf (accessed 26 February 2007).

5 Web site. http://www.ismp.org/PR/PediatricPharmacyGuidelines.htm (last accessed June 17, 2005)

6 Levine SR, for the Institute for Safe Medication Practices and the Pediatric Pharmacy Advocacy Group. Guidelines for preventing medication errors in pediatrics. J Pediatr Pharmacol Ther $2001 ; 6: 426-42$.

7 American Hospital Association. http://www.aha.org laccessed 13 February 2007)

8 Pathways for medication safety. http://www.medpathways.info/ medpathways/tools/tools.html (accessed 13 February 2007).

9 Berlin CM, McCarver DG, Notterman DA, et al. Prevention of medication errors in the pediatric inpatient setting. Pediatrics 1998;102:428-30.

10 Lannon CM, Coven BJ, Lane France F, et al. Principles of patient safety in pediatrics. Pediatrics 2001;107:1473-5.

11 Gorman RL, Bates BA, Benitz WE, et al. Prevention of medication errors in the pediatric inpatient setting. Pediatrics 2003;112:431-6.

12 National Initiative for Children's Healthcare Quality. http://www.nichq.org (accessed 13 February 2007).

13 National Quality Forum. http://www.qualityforum.org laccessed 13 February 2007).

14 National Quality Forum. Safe practices for better healthcare. 2003. (Document \#NQFCR-05-03.1

15 Massachusetts Coalition for the Prevention of Medical Errors. http:// www.macoalition.org/publications.shtml (accessed 13 February 2007).

16 Massachusetts Coalition for the Prevention of Medical Errors. MHA best practice recommendations to reduce medical errors. http:// www.macoalition.org/documents/Best_Practice_Medication_Errors.pdf (accessed 13 February 2007)

17 National Coordinating Council for Medication Error Reporting and Prevention. http://www.nccmerp.org (accessed 13 February 2007).

18 National Coordinating Council for Medication Error Reporting and Prevention. Council recommendation. http://www.nccmerp.org/councilRecs.html (accessed 13 February 2007).

19 Agency for Healthcare Research and Quality. http://www.ahrq.gov (accessed 13 February 2007)

20 Agency for Healthcare Research and Quality. Making health care safer: a critical analysis of patient safety practices. http://www.ahrq.gov/clinic/ptsafety/ (accessed 13 February 2007).

21 The Joint Commission. http://www.jcaho.org (accessed 13 February 2007).

22 The Joint Commission. http://www.jointcommission.org/PatientSafety/ NationalPatientSafetyGoals/ (accessed 26 February 2007).

23 Simpson JH, Lynch R, Grant J, et al. Reducing medication errors in the neonatal intensive care unit. Arch Dis Child Fetal Neonatal Ed 2004;89:F480-2.

24 France DJ, Cartwright J, Jones $\mathrm{V}$, et al. Improving pediatric chemotherapy safety through voluntary incident reporting: lessons from the field. J Pediatr Oncol Nurs 2004;21:200-6.

25 Petridou E, Kouri N, Vadala H, et al. Frequency and nature of recorded childhood immunization-related errors in Greece. J Toxicol Clin Toxicol 2004:42:273-6.

26 Cable G, Craft J. Agreement between pediatric medication orders and medication cardex. J Healthc Qual 2004;26:14-19.

27 Lehmann CU, Conner KG, Cox JM. Preventing provider errors: online total parenteral nutrition calculator. Pediatrics 2004;113:748-53.
28 Cimino MA, Kirschbaum MS, Brodsky L, et al. Assessing medication prescribing errors in pediatric intensive care units. Pediatr Crit Care Med 2004;5:124-32.

29 Cordero L, Kuehn L, Kumar RR, et al. Impact of computerized physician order entry on clinical practice in a newborn intensive care unit. J Perinatol 2004:24:88-93.

30 Potts AL, Barr FE, Gregory DF, et al. Computerized physician order entry and medication errors in a pediatric critical care unit. Pediatrics 2004;1 13:59-63.

31 Sangtawesin V, Kanjanapattanakul W, Srisan P, et al. Medication errors at Queen Sirikit National Institute of Child Health. J Med Assoc Thai 2003;86(Suppl 3):570-5.

32 Parshuram CS, Ng GY, Ho TK, et al. Discrepancies between ordered and delivered concentrations of opiate infusions in critical care. Crit Care Med 2003:31:2483-7.

33 King WJ, Paice N, Rangrej J, et al. The effect of computerized physician order entry on medication errors and adverse drug events in pediatric inpatients. Pediatrics 2003;112:506-9.

34 Fontan JE, Maneglier V, Nguyen VX, et al. Medication errors in hospitals: computerized unit dose drug dispensing system versus ward stock distribution system. Pharm World Sci 2003;25:112-17.

35 Holdsworth MT, Fichtl RE, Behta M, et al. Incidence and impact of adverse drug events in pediatric inpatients. Arch Pediatr Adolesc Med, 12003, 57:60-5.

36 Lesar TS. Tenfold medication dose prescribing errors. Ann Pharmacother 2002;36:1833-9

37 Derrough TF, Kitchin NR. Occurrence of adverse events following inadvertent administration of childhood vaccines. Vaccine 2002;21:53-9.

38 Frey B, Buettiker V, Hug Ml, et al. Does critical incident reporting contribute to medication error prevention? Eur J Pediatr 2002;161:594-9.

39 Kozer E, Scolnik D, Macpherson A, et al. Variables associated with medication errors in pediatric emergency medicine. Pediatrics 2002:110:737-42.

40 Pichon R, Zelger GL, Wacker $P$, et al. Analysis and quantification of prescribing and transcription errors in a paediatric oncology service. Pharm World Sci 2002;24:12-15.

41 Butte AJ, Shaw JS, Bernstein $\mathrm{H}$. Strict interpretation of vaccination guidelines with computerized algorithms and improper timing of administered doses. Pediatr Infect Dis J 2001;20:561-5

42 Kaushal R, Bates DW, Landrigan C, et al. Medication errors and adverse drug events in pediatric inpatients. JAMA 2001;285:2114-20.

43 Marino BL, Reinhardt K, Eichelberger WJ, et al. Prevalence of errors in a pediatric hospital medication system: implications for error proofing. Outcomes Manag Nurs Pract 2000;4:129-35.

44 Li SF, Lacher B, Crain EF. Acetaminophen and ibuprofen dosing by parents. Pediatr Emerg Care 2000;16:394-7.

45 Cote CJ, Notterman DA, Karl HW, et al. Adverse sedation events in pediatrics: a critical incident analysis of contributing factors. Pediatrics 2000;105:805-14.

46 Losek JD. Acetaminophen dose accuracy and pediatric emergency care. Pediatr Emerg Care 2004;20:285-8.

47 Upperman JS, Staley P, Friend K, et al. The impact of hospitalwide computerized physician order entry on medical errors in a pediatric hospital. Pediatr Surg 2005;40:57-9

48 Cowley E, Williams R, Cousins D. Medication errors in children: a descriptive summary of medication error reports submitted to the United States Pharmacopeia. Curr Ther Res Clin Ex 2001;62:627-40.

49 Farrar K, Caldwell NA, Robertson J, et al. Use of structured paediatricprescribing screens to reduce the risk of medication errors in the care of children. Br J Healthcare Comput Info Manage 2003;20:25-7.

50 Slonim AD, LaFleur B, Ahmed W, et al. Hospital-reported medical errors in children. Pediatrics 2003;111:617-21.

51 Feikema SM, Klevens RM, Washington ML, et al. Extraimmunization among US children. JAMA 2000;283:1311-17.

52 Goldman RD, Scolnik D. Underdosing of acetaminophen by parents and emergency department utilization. Pediatr Emerg Care 2004:20:89-93.

53 McErlean MA, Bartfield JM, Kennedy DA, et al. Home antipyretic use in children brought to the emergency department. Pediatr Emerg Care 2001;17:249-51.

54 McPhillips $\mathrm{H}$, Stille C, Smith $\mathrm{D}$, et al. Methodological challenges in describing medication dosing errors in children. In: Henriksen K, Battles J, Marks E, Lewin DI, eds. Advances in patient safety: from research to implementation. Vol 2. Concepts and methodology. Rockville, MD: Agency for Healthcare Research and Quality, 2005:213-23.

55 McPhillips HA, Stille CJ, Smith D, et al. Medication dosing errors in outpatient pediatrics. J Pediatrics 2005;147:761-7. 